DOI: http://doi.org/10.21698/simi.2018.ab40

\title{
SAMPLE PREPARATION USING GERSTEL SPME MULTIFIBER EXCHANGE (MFX)
}

Luminita Barbu, Emilia Teaca

Apa Nova Company, 60A Tunari Street, Stefan cel Mare building, 6-9 floor, Bucharest, luminita.barbu@,veolia.com, Romania

Keywords: $M F X$, sample preparation, $S P M E$

\section{Introduction}

Solid phase micro extraction (SPME) was developed to address to need to facilitate rapid sample preparation in the laboratory. SPME fiber consists of a small amount of the extraction liquid phase deposited on a solid support.

Solid phase micro extraction is based on the distribution of the analyte between the sample and the absorbent layer of the fiber and it is considered that the separation is complete when the analyte concentration has reached distribution equilibrium between the sample matrix and the fibber coating. The analytical basis for quantification through this technique is linear dependence between the amount of analyte extracted onto the coating and the analyte concentration in the sample under well-established operating conditions.

\section{Materials and methods}

Pentachloro-nitro benzene $(5000 \mathrm{ug} / \mathrm{mL}$ in acetone) was obtained from Ultra Scientific Analytical Solutions. All chemicals were of analytical grade with purity above $99 \%$. Fibers PDMS with coating thickness $100 \mu \mathrm{m}$, were purchased from Supelco. All experiments were performed on a GC (7890A, Agilent Technologies) with a micro electron capture detector and mass selective detector (5975C Agilent Technologies), a Multi-Mode split/split less inlet used in the split less mode, and a MultiPurpose Sampler with SPME capability (MPS 2, Gerstel). The whole analytical procedure was controlled with the program MSDChemStation (Agilent Technologies) and Maestro (Gerstel).

\section{Results and conclusions}

The parameters that affect the SPME process were evaluated and optimized: Final and initial conditioning of the fiber, Incubation time, Incubation temperature, Extraction time and Desorption time.

After optimization, the time is reduced by approximately $15 \%$ (Figure 1 ).

Using the overlay option of the work steps from Maestro soft the time is reduced by $25 \%$ (Figure 2).

Comparing the LLE with SPME, it is found that the time needed to process a sample by SPME is 3 times smaller than by LLE (Figure 3 ).

Good recovery performance and reproducibility are found for SPME technique. 


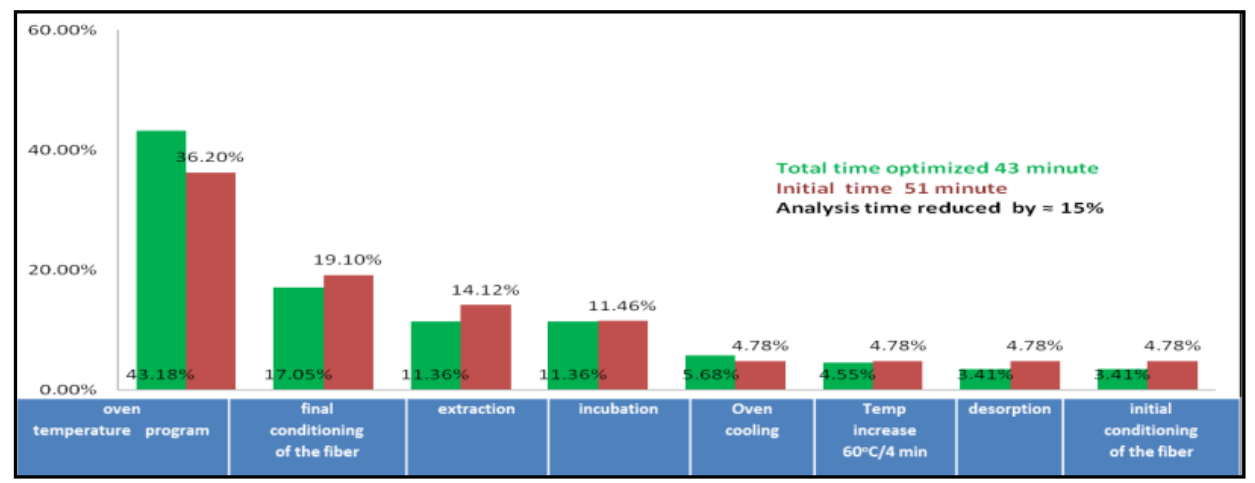

Figure 1. Operational parameters optimization

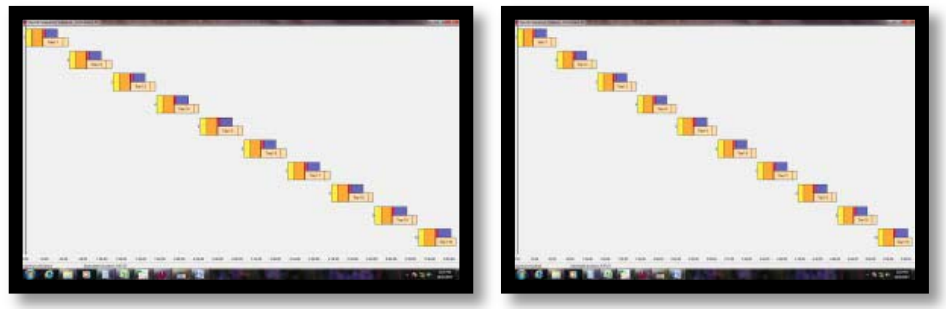

Figure 2. Prep-ahead option of Maestro soft (with and no option)

\begin{tabular}{|c|c|c|c|c|c|c|c|c|c|}
\hline \multicolumn{10}{|c|}{ GC - LLE $120 \mathrm{~min}$} \\
\hline \multicolumn{3}{|c|}{ LLE } & \multicolumn{4}{|c|}{ anhydrifying } & $\begin{array}{c}\text { evaporatio } \\
n\end{array}$ & \multicolumn{2}{|c|}{ GC analysis } \\
\hline \multicolumn{10}{|c|}{ sample 1} \\
\hline \multicolumn{10}{|c|}{ GC - SPME $43 \mathrm{~min}$} \\
\hline incub & extr & des & GC analysis & incub & extr des & GC analysis & incub & des & GC analysis \\
\hline \multicolumn{4}{|c|}{ sample 1} & \multicolumn{3}{|c|}{ sample 2} & \multicolumn{3}{|c|}{ sample 3} \\
\hline
\end{tabular}

Figure 3. Working time for GC-LLE and GC-SPME

Table 1. Recovery efficiency and reproducibility (average of 7 replicates)

\begin{tabular}{|c|c|c|c|c|}
\hline Technique & Added, $\mu \mathrm{g}$ & Found, $\mu \mathrm{g}$ & $\begin{array}{c}\text { Reproducibility, } \\
(\mathrm{CV} \%)\end{array}$ & $\begin{array}{c}\text { Recovery } \\
\text { efficiency, } \%\end{array}$ \\
\hline LLE & \multirow{2}{*}{20} & 13.64 & 18.38 & 68.19 \\
\cline { 3 - 5 } SPME & 18.38 & 3.01 & 92.10 \\
\hline
\end{tabular}

Advantages of the SPME technique:

- Less analysis time vs. LLE with over $75 \%$

- Recovery yield increased by $>50 \%$ compared to LLE

- The reproducibility of the entire analysis process is about 6 times better than LLE

- Increased sensitivity compared to LLE

- Required sample volume reduced compared to LLE (from 1L to $10 \mathrm{~mL}$ )

- The absence of organic solvents with environmental and health impacts. 